EPJ Web of Conferences 86,00060 (2015)

DOI: $10.1051 /$ epjconf/ 20158600060

(C) Owned by the authors, published by EDP Sciences, 2015

\title{
Statistical model calculations of pre-scission neutron multiplicity for the heavy ion induced fusion-fission reactions with actinide target ${ }^{232} \mathrm{Th}$
}

\author{
Meenu Thakur ${ }^{1}$,a , B.R. Behera ${ }^{1}$, Maninder Kaur ${ }^{1}$, Santanu Pal ${ }^{2}$, P. Sugathan ${ }^{3}$, and Akhil Jhingan ${ }^{3}$ \\ ${ }^{1}$ Department of Physics, Panjab University, Chandigarh 160014, India \\ ${ }^{2}$ CS - 6/1, Golf Green, Kolkata 700095, India (Formerly with VECC, Kolkata) \\ ${ }^{3}$ Inter University Accelerator Centre, Aruna Asaf Ali Marg, New Delhi 110067, India
}

\begin{abstract}
The reaction mechanism of ${ }^{19} \mathrm{~F}+{ }^{232} \mathrm{Th}$ and ${ }^{28} \mathrm{Si}+{ }^{232} \mathrm{Th}$ systems populating the near-super-heavy compound nuclei ${ }^{251} \mathrm{Es}$ and ${ }^{260} \mathrm{Rf}$ respectively are investigated using neutron multiplicity as a probe. The prescission neutron multiplicities of these compound nuclei are calculated at different excitation energies using a statistical model code. These calculations are performed using the Bohr-Wheeler transition state fission width as well as the dissipative dynamical fission width based on the Kramers' prescription. For ${ }^{19} \mathrm{~F}+{ }^{232} \mathrm{Th}$ system, the measured yield of pre-scission is compared with the statistical model calculations for the decay of a compound nucleus in the excitation energy range of $54-90 \mathrm{MeV}$. The comparison between the measured and the calculated values indicates that the Bohr-Wheeler fission width underestimates the pre-scission neutron yield and a large amount of dissipation strength is required to reproduce the experimental pre-scission neutron multiplicities. The excitation energy dependence of the fitted values of the dissipation coefficient is also discussed. In addition, exploratory statistical model calculations of pre-scission neutron multiplicity for the ${ }^{28} \mathrm{Si}+{ }^{232} \mathrm{Th}$ system are presented in the above range of excitation energy.
\end{abstract}

\section{Introduction}

The heavy elements beyond nobelium have been successfully produced by fusion of two lighter nuclei using two methods: Actinide based $4 \mathrm{n}$ and $5 \mathrm{n}$ reactions at excitation energies in the range of $40-50 \mathrm{MeV}$ and $\mathrm{Pb} / \mathrm{Bi}$ based reactions in the range of 10-20 MeV. Experimental efforts to produce the super-heavy nuclei followed the predictions of an island of super-heavy nuclei having enhanced stability due to shell effects [1]. Such experiments are extremely challenging as the formation of heavy and superheavy evaporation residues (ERs) are suppressed not only by equilibrium fission, but also by quasi-fission. The main objective in the super-heavy element production is to identify those variables that hinder compound nucleus (CN) formation. This problem can be better addressed by measuring the characteristics of the quasi-fission events. The properties such as entrance channel, in particular the entrance channel mass-asymmetry (closely related to the product of projectile and target atomic number, $\mathrm{Z}_{p} \mathrm{Z}_{t}$ ) and the deformation of colliding nuclei [2], play a major role in the reaction dynamics of quasi-fission process. The reactions with deformed nuclei are pushed by side collisions to higher excitation energies (4n- and 5n-channels), whereas reactions carried by the closed-shell nuclei are kept at low excitation energies (1n- and 2n-channels). With these motivations, we have planned to study the reaction mechanism of ${ }^{19} \mathrm{~F}+{ }^{232} \mathrm{Th}$ and ${ }^{28} \mathrm{Si}+{ }^{232} \mathrm{Th}$ systems

${ }^{\mathrm{a}} \mathrm{e}$-mail: meenu.thakuroct $@$ gmail.com populating near-super-heavy $\mathrm{CN}{ }^{251} \mathrm{Es}$ and ${ }^{260} \mathrm{Rf}$ respectively using neutron multiplicity as a probe.

The importance of neutrons as a probe for fusionfission dynamics was first pointed out in a series of earlier pioneering works (e.g. see [3-6]). These works established that fission becomes a slow process in comparison to the transition-state theory of Bohr and Wheeler [7] beyond a certain excitation energy for heavy compound nuclei. This fission hindrance is usually attributed to a dissipative dynamics of nuclear fission. In the next section, we present results of statistical model calculation in order to explore the role of dissipation in pre-scission neutron multiplicities of ${ }^{19} \mathrm{~F}+{ }^{232} \mathrm{Th}$ and ${ }^{28} \mathrm{Si}+{ }^{232} \mathrm{Th}$ systems.

\section{Statistical model calculations}

Since the beginning of nuclear science, the statistical concepts and models have been used to understand the different nuclear reaction mechanisms. As nucleus is a many body complex system, even a small perturbation in terms of excitation energy may result in many different configurations. Therefore, statistical methods are essential for the comprehension and prediction of many nuclear phenomena in such a complex situation. The heavy ion induced fusion reaction involves the $\mathrm{CN}$ formation with very high excitation energy and angular momenta which undergoes rapid decay to its ground state via various exit channels. The decay of the $\mathrm{CN}$ is successfully described by the statistical models. These models assume that all possibilities 
for decay are intrinsically equally likely and are governed by factors such as the density of the final states and barrier penetration factors. Further, the probability for a particular decay to occur is inversely proportional to the total number of possible decays. The presence of centrifugal, coulomb or other type of potential barrier reduces this population probability. These statistical assumptions, when combined with the conservation laws and principle of detailed balance leads to a statistical model which predicts the average cross-sections. These models can be used to verify the reaction mechanisms and to search for non-statistical aspects of nuclear structure at high excitation energies and angular momenta. In fusion-fission reactions, the formed CN decays via two major channels: particle emission and fission. The heavy $\mathrm{CN}$ splits into two fragments in the fission process. The light particles (proton, neutron and $\alpha$ ) and gamma quanta are emitted during the passage of the intermediate composite system from equilibrium deformation to saddle point and from saddle point to scission point. These particles emitted before (after) scission point are called pre (post)-scission particles. In a statistical model, the emission of these light particles (proton, neutron and $\alpha$ ) and gamma rays have been considered as the decay channels for the excited $\mathrm{CN}$ in addition to the fission channel.

It may however be pointed out here that experimentally determined pre-scission particle multiplicities may contain contributions from particles emitted from the fission fragments during their accelerating phase [3-6]. This contribution is expected to be small when the life-time of the compound nucleus is large compared to the time scale of the accelerating phase, and conversely, the contribution can be large for compound nuclei with small fission barriers and/or at high excitation energies [5].

Important ingredients used in the statistical model calculations are: a.) the nuclear density of states, b.) the Bohr-Wheeler (BW) fission width and c.) the Kramers' fission width. The nuclear level density $\rho$ (E) plays a central role in the theoretical modelling of decay of hot $\mathrm{CN}$. The standard form of the level density formula [7] can be written as

$$
\rho\left(E^{*}, \ell\right)=\frac{2 \ell+1}{24}\left[\frac{\hbar^{2}}{2 \mathfrak{J}}\right]^{3 / 2} \frac{\sqrt{a}}{E^{* 2}} \exp \left(2 \sqrt{a E^{*}}\right)
$$

where $\ell$ is the compound nuclear spin, $\mathfrak{J}$ is the rigid body moment of inertia of the $\mathrm{CN}$ and the quantity ' $a$ ' is called the level density parameter which, according to the Fermi gas model, is related to the nuclear temperature ' $T$ ' by the equation $E^{*}=a T^{2}$. With the above description of the density of states and the level density parameter, BW fission width can be calculated from the following equation [8]

$$
\Gamma_{B W}=\frac{1}{2 \pi \rho\left(E^{*}\right)} \int_{0}^{E^{*}-V_{B}} d \epsilon \rho^{*}\left(E^{*}-V_{B}-\epsilon\right)
$$

where $\epsilon$ is the kinetic energy and $V_{B}$ is the fission barrier height. This derivation for the fission width is valid only if the number of states in the transition state is sufficiently large compared to unity. It corresponds to the conditions

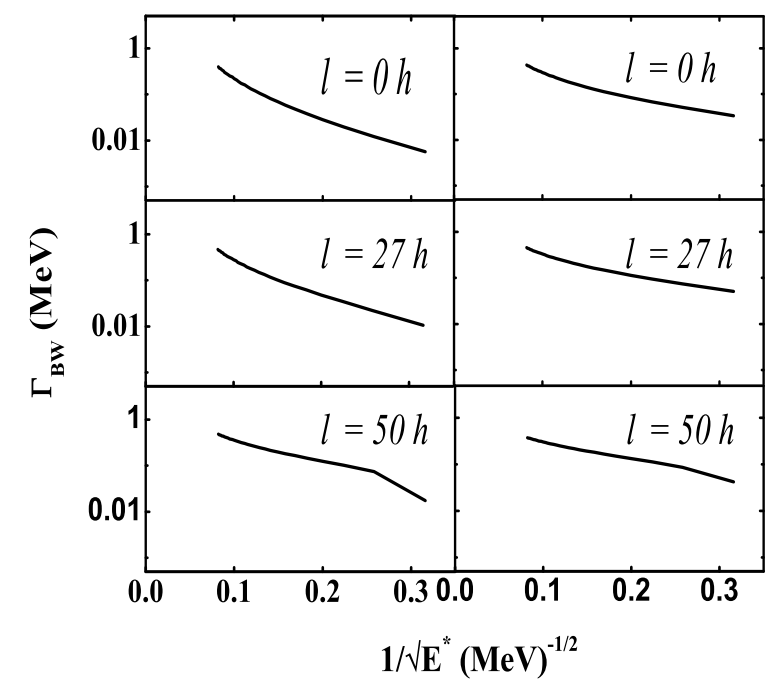

Figure 1. The calculated BW fission width for the $\mathrm{CN}^{251} \mathrm{Es}$ (left side) and ${ }^{260} \mathrm{Rf}$ (right side) versus $1 / \sqrt{E^{*}}$.

under which the statistical mechanics can be applied for fission. On the other hand, when the excitation energy exceeds $V_{B}$ by a small amount or falls below $V_{B}$, the specific quantum-mechanical tunneling effect becomes important. Also, the density of states $\rho^{*}$ is different from $\rho$ in a sense that it does not contain the degree of freedom associated with the fission itself. The calculated BW fission widths for both the $\mathrm{CN}^{251} \mathrm{Es}$ and ${ }^{260} \mathrm{Rf}$ at different excitation energies and $\ell$ values are shown in figure 1 . In fusion-fission reactions, it is found that $\mathrm{BW}$ fission width grossly underestimates the measured pre-scission neutron yield at all energies. This may be due to the onset of dynamical effects, which delays the fission process. Therefore, the nuclear friction $\left(\gamma=\beta / 2 \omega_{s}\right)$ is considered necessary in order to explain the data on pre-scission light particles and gamma rays. Hence, BW fission width replaced by Kramers' width with different ' $\beta$ ' values. The Kramers' fission width [9] is given as:

$$
\Gamma_{K}=\frac{\hbar \omega_{g}}{2 \pi} \exp \left(-V_{B} / T\right)\left[\sqrt{1+\left(\frac{\beta}{2 \omega_{s}}\right)^{2}}-\frac{\beta}{2 \omega_{s}}\right]
$$

where $\beta$ is the reduced dissipation strength, $V_{B}$ is the fission barrier height, $\omega_{g}$ and $\omega_{s}$ are the frequencies of the harmonic oscillators at the ground state and at the saddle configurations.

By using the widths of all the decay modes explained above, the time evolution of a $\mathrm{CN}$ was followed in the statistical model code until either fission occurs or an ER is formed. The decay of a $\mathrm{CN}$ is followed using MonteCarlo method. The effect of a transient time to build up the stationary fission width is also included in the calculation by using a time-dependent fission width $[10,11]$. The Monte-Carlo procedure enroutes the fission in a certain interval, the corresponding trajectory is then counted as a fission event. If the procedure does not select the fission channel at a certain interval but selects a particle/gamma 
emission, the intrinsic energy and angular momentum is recalculated for the daughter nucleus and the procedure is followed until the intrinsic energy becomes less than the particle binding energy, $\mathrm{U}<\min \left(B_{n}, B_{p}, B_{\alpha}, B_{f}\right)$. In this case, evaporation residue is counted as the end product. Using the above mentioned approach, the number of light particles as well as photons are counted for each fission event. In addition to this for a fission event, the number of particles emitted during saddle-to-scission transition is also calculated $[12,13]$. The computations have been performed over a large ensemble and the multiplicities of the pre-scission neutrons are thus obtained from the statistical model code. The fusion spin distribution (CN spin distribution) as used as the input to the model in the calculations. In the present work, we have used the spin distribution of the $\mathrm{CN}$ from the empirical formula given in [14].

\section{Results and discussion}

In this work, we have performed statistical model calculations for two systems ${ }^{19} \mathrm{~F}+{ }^{232} \mathrm{Th}$ and ${ }^{28} \mathrm{Si}+{ }^{232} \mathrm{Th}$ populating near-super-heavy $\mathrm{CN}^{251} \mathrm{Es}$ and ${ }^{260} \mathrm{Rf}$ respectively in same excitation energy range of 54-90 MeV using a statistical model code [15]. The calculated pre-scission neutron multiplicity values for both the systems using the BW and Kramers' width with the dissipation strength value $(\beta)$ are given in table 1. The excitation functions of pre-scission neutron multiplicity for both the systems are shown in figure 2 and figure 4 . Figure 2 shows the results obtained for the ${ }^{19} \mathrm{~F}+{ }^{232} \mathrm{Th}$ system along with the experimental data which was first reported in [3] and was later made available in tabular form in [4]. We first note that the BW fission width severely underestimates the experimental multiplicities. Therefore, value of ' $\beta$ ' is increased to fit the experimental data.

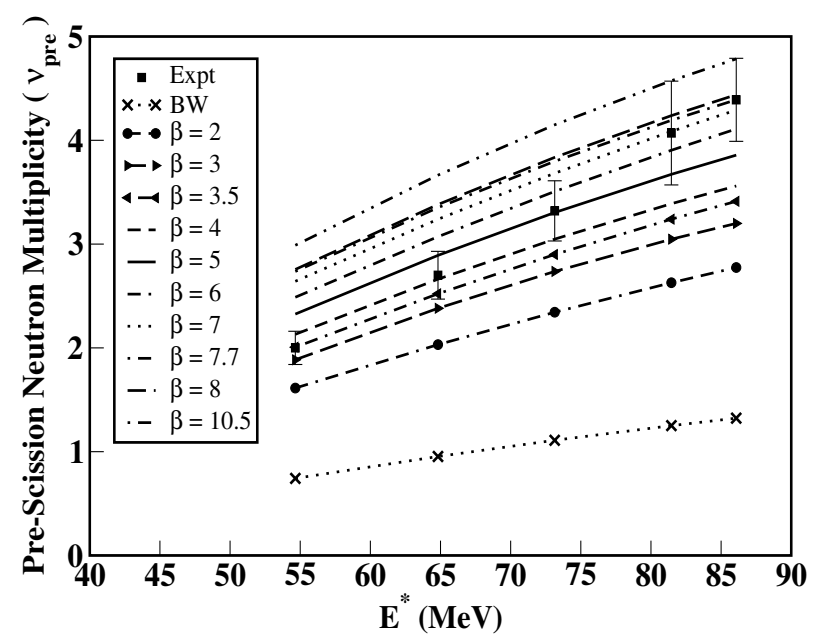

Figure 2. Pre-Scission neutron multiplicities calculated using BW width and Kramers' width for the ${ }^{19} \mathrm{~F}+{ }^{232} \mathrm{Th}$ system.

Further, we are also reporting here the calculated dissipation strength for ${ }^{19} \mathrm{~F}+{ }^{232} \mathrm{Th}$ system by reproducing its experimental data of pre-scission neutron multiplicity

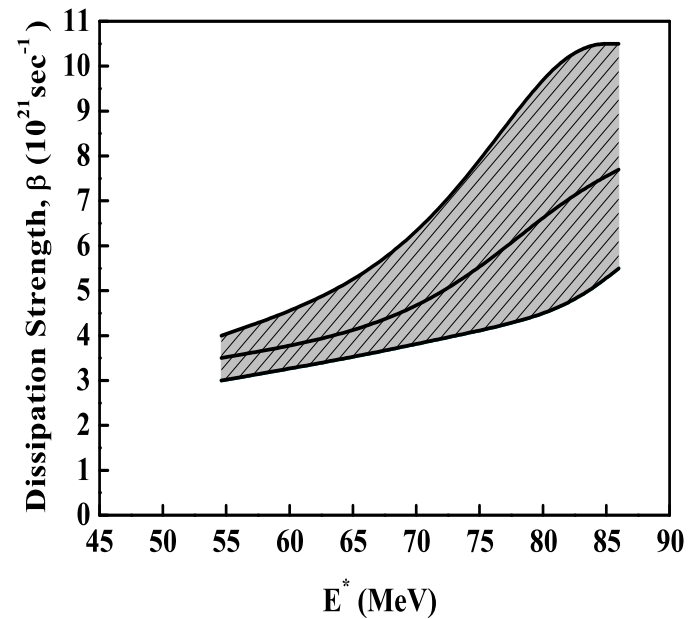

Figure 3. The uncertainty in ' $\beta$ ' for ${ }^{19} \mathrm{~F}+{ }^{232} \mathrm{Th}$ system. The solid line indicates the best fit ' $\beta$ ' values obtained at each excitation energy.

using a statistical model code in the same excitation energy range. The fitted value of ' $\beta$ ' increases with the excitation energy as shown in figure 3 . The hatched area represents the uncertainty in value of ' $\beta$ ' arises due to the experimental error bars. Along with this we are also presenting the exploratory statistical model calculation of prescission neutron multiplicity for the ${ }^{28} \mathrm{Si}+{ }^{232} \mathrm{Th}$ system in the same excitation energy range. Since no experimental data exists for pre-scission neutron multiplicity for ${ }^{28} \mathrm{Si}+$ ${ }^{232} \mathrm{Th}$ system in a wider excitation energy range, we have planned to carry out this experiment in near future.

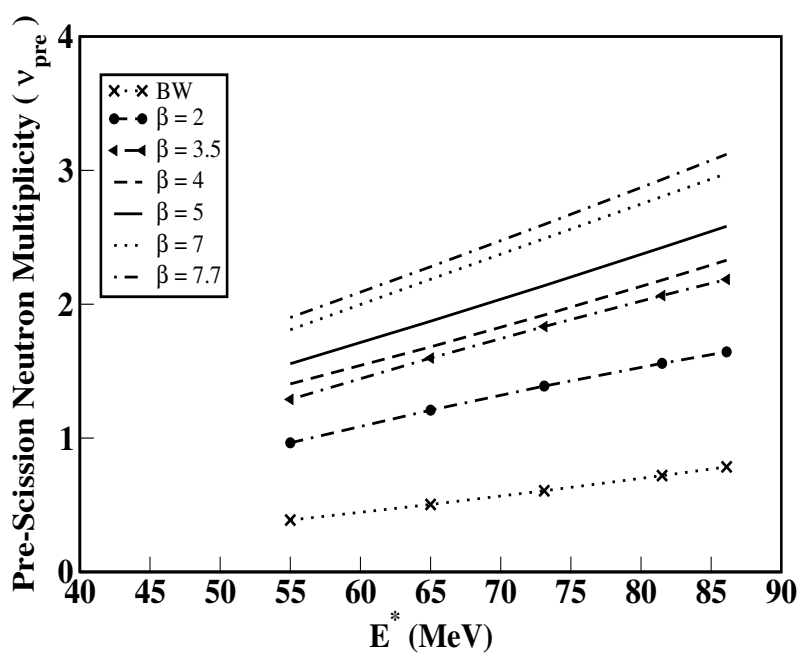

Figure 4. Pre-Scission neutron multiplicities calculated using BW width and Kramers' width for the ${ }^{28} \mathrm{Si}+{ }^{232} \mathrm{Th}$ system.

Figure 4 indicates only the exploratory calculations of prescission neutron multiplicity for ${ }^{28} \mathrm{Si}+{ }^{232} \mathrm{Th}$ system. This study provides us an estimate of pre-scission neutron multiplicities which one might expect from the experimental results. We observe that the pre-scission neutron multiplicity reduces with increase in projectile mass as we go 
Table 1. The pre-scission neutron multiplicity $\left(v_{\text {pre }}\right)$ at different excitation energies calculated using the Bohr-Wheeler width and the Kramers' modified width with the dissipation strength value $(\beta)$.

\begin{tabular}{|c|c|c|c|c|c|c|c|c|c|c|}
\hline Systems & $\begin{array}{c}\mathrm{E}^{*} \\
(\mathrm{MeV})\end{array}$ & $\begin{array}{c}v_{\text {pre }} \\
(\mathrm{BW})\end{array}$ & $\begin{array}{c}v_{\text {pre }} \\
(\beta=2)\end{array}$ & $\begin{array}{c}v_{\text {pre }} \\
(\beta=3.5)\end{array}$ & $\begin{array}{c}v_{\text {pre }} \\
(\beta=4)\end{array}$ & $\begin{array}{c}v_{\text {pre }} \\
(\beta=5)\end{array}$ & $\begin{array}{c}v_{\text {pre }} \\
(\beta=7)\end{array}$ & $\begin{array}{c}v_{\text {pre }} \\
(\beta=7.7)\end{array}$ & $\begin{array}{c}v_{\text {pre }} \\
(\text { Exp })\end{array}$ & Fitted $\beta$ \\
\hline \multirow{3}{*}{${ }^{19} \mathrm{~F}+{ }^{232} \mathrm{Th}$} & 54.6 & 0.744 & 1.610 & 2.010 & 2.130 & 2.330 & 2.640 & 2.740 & $2.00 \pm 0.16$ & $3.5(+0.5,-0.5)$ \\
& 64.8 & 0.949 & 2.040 & 2.510 & 2.670 & 2.880 & 3.240 & 3.340 & $2.70 \pm 0.23$ & $4.0(+1.0,-0.5)$ \\
& 73.1 & 1.114 & 2.340 & 2.910 & 3.030 & 3.310 & 3.690 & 3.810 & $3.32 \pm 0.29$ & $5.0(+2.0,-1.0)$ \\
& 81.5 & 1.251 & 2.620 & 3.240 & 3.410 & 3.680 & 4.069 & 4.190 & $4.07 \pm 0.50$ & $7.0(+3.5,-2.5)$ \\
& 86.0 & 1.322 & 2.780 & 3.410 & 3.550 & 3.850 & 4.300 & 4.390 & $4.39 \pm 0.40$ & $7.7(+2.8,-2.2)$ \\
\hline \multirow{3}{*}{$\mathrm{2} \mathrm{Si}+{ }^{232} \mathrm{Th}$} & 54.6 & 0.381 & 0.959 & 1.284 & 1.408 & 1.551 & 1.804 & 1.896 & & \\
& 64.8 & 0.519 & 1.226 & 1.611 & 1.672 & 1.882 & 2.204 & 2.295 & No Data & \\
& 73.1 & 0.607 & 1.376 & 1.827 & 1.924 & 2.142 & 2.472 & 2.583 & available & \\
& 81.5 & 0.692 & 1.552 & 2.053 & 2.187 & 2.402 & 2.809 & 2.932 & & \\
\end{tabular}

from ${ }^{19} \mathrm{~F}$ to ${ }^{28} \mathrm{Si}$. This evidently reflects the effect of lowering of fission barrier at higher compound nuclear spin populated by heavier projectiles. The experimental multiplicities may however be still smaller for heavier projectiles due to quasi-fission. We aim to investigate these features in our planned experiments in near future.

At the end, we point out that the contribution of neutrons emitted from the fission fragments during their accelerating phase has not been considered in the above calculations. This contribution can be large for highly fissile compound nuclei at high excitations and is included in the experimentally obtained pre-scission multiplicities [5]. Therefore, the "true" pre-scission multiplicity can be smaller than those deduced experimentally. Consequently, the best-fit values of the dissipation strength could be lower than the values reported in the present work.

\section{Acknowledgement}

The financial support from the University Grants Commission (UGC) to one of the authors (M. T.) is gratefully acknowledged.

\section{References}

[1] P. Armbruster, C. R. Phys. 4, 571 (2003)

[2] J.P. Blocki, H. Feldmeier and W.J. Swiatecki, Nucl. Phys. A 459, 145 (1986)
[3] D.J. Hinde, R.J. Charity, G.S. Foote, J.R. Leigh, J.O. Newton, S. Ogaza, and A. Chatterjee, Phys. Rev. Lett. 52, 986 (1984); Phys. Rev. Lett. 53, 2275 (1984)

[4] J.O. Newton, D.J. Hinde, R.J. Charity, J.R. Leigh, J.J.M. Bokhorst, A. Chatterjee, G.S. Foote, and S. Ogaza, Nucl. Phys. A 483, 126 (1988)

[5] D.J. Hinde, D. Hilscher, H. Rossner, B. Gebauer, M. Lehmann, and M. Wilpert, Phys. Rev. C 45, 1229 (1992)

[6] D.J. Hinde, Nucl. Phys. A 553, 255 (1993)

[7] A. Bohr and B.R. Mottelson, Nuclear structure Vol. I (Benjamin Press, New York, 1969)

[8] N. Bohr and J.A. Wheeler, Phys. Rev. 56, 426 (1939)

[9] H.A. Kramers, Physica (Amsterdam) 7, 284 (1940)

[10] P. Grangé, Li Jun-Qing, and H.A. Weidenmüller, Phys. Rev. C 27, 2063 (1983)

[11] K.H. Bhatt, P. Grangé, and B. Hiller, Phys. Rev. C 33, 954 (1986)

[12] H. Hofmann and J.R. Nix, Phys. Lett. B 122, 117 (1983)

[13] P. Grangé, S. Hassani, H.A. Weidenmüller, A. Gavron, J.R. Nix, and A.J. Sierk, Phys. Rev. C 34, 209 (1986)

[14] P. Fröbrich and I.I. Gontchar, Phys. Rep. 292, 131 (1998)

[15] Jhilam Sadhukhan and Santanu Pal, Phys. Rev. C 78, 011603 (R) (2008); Phys. Rev. C 79, 019901(E) (2009) 